\title{
Intravenous Anesthetic Protects Hepatocyte from Reactive Oxygen Species-Induced Cellular Apoptosis during Liver Transplantation In Vivo
}

\author{
Weifeng Yao $\left(\mathbb{D},{ }^{1}\right.$ Xue Han, ${ }^{2}$ Yihan Zhang $\left(\mathbb{D},{ }^{1}\right.$ Jianqiang Guan, ${ }^{1}$ Mian Ge, ${ }^{1}$ Chaojin Chen, \\ Shan Wu, ${ }^{1}$ Jiaxin Chen, ${ }^{1}$ Gangjian Luo, ${ }^{1}$ Pinjie Huang $\left({ }^{1},{ }^{1}\right.$ and Ziqing Hei $\oplus^{1}$ \\ ${ }^{1}$ Department of Anesthesiology, Third Affiliated Hospital, Sun Yat-sen University, Guangzhou, Guangdong 510630, China \\ ${ }^{2}$ Department of Anesthesiology, Sun Yat-sen Memorial Hospital, Sun Yat-sen University, Guangzhou 510000, China \\ Correspondence should be addressed to Pinjie Huang; hpjie@126.com and Ziqing Hei; heiziqing@sina.com
}

Received 22 May 2018; Accepted 28 August 2018; Published 28 October 2018

Academic Editor: Aldrin V. Gomes

Copyright (C) 2018 Weifeng Yao et al. This is an open access article distributed under the Creative Commons Attribution License, which permits unrestricted use, distribution, and reproduction in any medium, provided the original work is properly cited.

\begin{abstract}
Background. Liver transplantation leads to liver ischemia/reperfusion (I/R) injury, resulting in early graft dysfunction and failure. Exacerbations of oxidative stress and inflammatory response are key processes in the development of liver I/R injury. Intravenous anesthetic propofol potent effects on free radical scavenging and protects livers against I/R injury. However, the role and mechanism of propofol-mediated hepatic protection in liver transplantation is poorly understood. The aim of this study was to evaluate the role of propofol postconditioning in the liver I/R injury after liver transplantation. Methods. Forty-eight rats were randomly divided into six groups: rats receiving either sham operation or orthotopic autologous liver transplantation (OALT) in the absence or presence of propofol (high dose and low dose) postconditioning or intralipid control or VAS2870 (Nox2 special inhibitor). Eight hours after OALT or sham operation, parameters of organ injury, oxidative stress, inflammation, and NADPHassociated proteins were assessed. Results. After OALT, severe liver pathological injury was observed that was associated with increases of serum AST and ALT, which were attenuated by propofol postconditioning. In addition, especially high dose of propofol postconditioning reduced TNF- $\alpha$, IL- $1 \beta$, IL-6, TLR4, and NF- $\kappa$ B inflammatory pathway, accompanied with decrease of neutrophil elastase activity, MPO activity, 8-isoprotane, $\mathrm{p} 47^{\text {phox }}$ and gp91 ${ }^{\text {phox }}$ protein expressions, and increase of SOD activity. Inhibition of Nox 2 by VAS2870 conferred similar protective effects in liver transplantation. Conclusion. Liver transplantation leads to severe inflammation and oxidative stress with NADPH oxidase activation. Propofol postconditioning reduces liver I/R injury after liver transplantation partly via inhibiting NADPH oxidase Nox2 and the subsequent inflammation and oxidative stress.
\end{abstract}

\section{Introduction}

Liver transplantation has become the effective surgical treatment for patients with end-stage liver disease [1]. Liver ischemia reperfusion (I/R) injury is a severe postoperative complication during the early period after transplantation. It leads to early graft dysfunction and failure, which further results in acute and chronic rejection and irreversible death [2]. Characterized by uncontrolled inflammatory response, liver I/R injury promotes hypoxic hepatocyte reoxygenation and reactive oxygen species (ROS) formation, which result in neutrophil infiltration, robust ROS generation, and ultimately programmed death of hepatocytes [3]. To date, the mechanisms accounted for liver injury, especially I/R injury during liver transplantation, are complicated and remained unclear; strategies for preventing I/R injury are still lacking. Hence, seeking protective intervention of $\mathrm{I} / \mathrm{R}$ injury during perioperative period is regarded urgent and profound scientific significance and important clinical applications.

Propofol (2,6-diisopropylphenol) is widely used for anesthesia induction and maintenance during perioperation and sedation in intensive care unit (ICU) patients [4]. In addition to its clinical usages, propofol exerts antiinflammatory and antioxidative effectiveness basing on the chemical group phenol [5]. In our previous study, we found that propofol pretreatment could attenuate pulmonary 
oxidative stress induced by liver transplantation through activating Nrf2 nuclear translocation and upregulating its downstream of HO-1 antioxidant enzyme formation $[6,7]$. However, in most clinical situations, propofol pretreatment is not feasible while treatment at the onset or after induction of reperfusion, termed as postconditioning, is more applicable [8], which has been proven as a promising therapeutic strategy against ischemia/reperfusion damage. Whether propofol postconditioning is crucial in reducing oxidative stress in liver I/R injury and the underline mechanism remains unknown.

Nicotinamide adenine dinucleotide phosphate (NADPH) oxidase (Noxs) is one of the major sources of cellular ROS, which has been identified to play an important role in liver I/R injury [9]. Nox2 and Nox4 are two predominant Nox isoforms existing in hepatocytes in liver parenchyma [10]. Nox2-deficient mice showed lower mortality rate than wild type group when subjected to hepatic I/R injury. Whether Nox2 is correlated to liver transplantationinduced hepatic oxidative stress and contributed to the antioxidant property of propofol need to be verified.

Therefore, the current study observed the effects of propofol postconditioning on liver I/R injury induced by liver transplantation and further explored the potential mechanism whether the protective effects provided by propofol postconditioning are associated with Nox2-related oxidative stress pathway.

\section{Materials and Methods}

2.1. Experimental Protocols. Male Sprague-Dawley rats (220-250 g, 8 weeks) obtained from Medical Experimental Animal Center of Guangdong Province (Guangzhou, China) were housed in the animal room of Zhongshan Medical School (Guangzhou, China). Rats were fasted for 8 hours prior to the study but were allowed to access tap water ad libitum. All the animal care and research protocols were approved by the Institutional Animal Care and Use Committee of Sun Yat-sen University (Guangzhou, China) and performed in accordance with National Institutes of Health guidelines for the use of experimental animals.

The orthotopic autologous liver transplantation (OALT) model was carried out according to our previous study $[11,12]$. Rats were randomly divided into six groups $(n=8)$ as follows: sham-operated control (sham) and OALT, OALT treated with intralipid (OALT + INT), OALT treated with high dose of propofol (OALT + HPro), OALT treated with low dose of propofol (OALT + LPro), and OALT treated with VAS2870 (OALT + VAS). High dose $(40 \mathrm{mg} / \mathrm{kg} / \mathrm{h})$ or low dose $(20 \mathrm{mg} / \mathrm{kg} / \mathrm{h})$ of propofol [13] or the same volume of intralipid was administrated continuous via tail vein for $30 \mathrm{~min}$ at the onset of reperfusion. Some of the rats were treated with specific Nox2 inhibitor VAS2870 $(2 \mathrm{mg} / \mathrm{kg}$, Sigma, USA) [14] intravenously after reperfusion.

2.2. Sample Harvest. Blood and liver samples were harvested eight hours after reperfusion. Under general anesthesia, animals were euthanized by a lethal injection of sodium pentobarbital. The blood was collected from carotid artery into heparinized tubes and then centrifuged for $15 \mathrm{~min}$ at $2000 \mathrm{~g}$ $\left(4^{\circ} \mathrm{C}\right)$. The supernatants were collected and stored at $-80^{\circ} \mathrm{C}$ until measurement. Median hepatic lobes were immediately and promptly taken out (about $0.5 \mathrm{~cm}^{3}$ ), washed in cold saline, fixed in $10 \%$ formalin solution, dehydrated in ascending grades of alcohol, and then embedded in paraffin. The residual parts of liver tissue were harvested and stored at $-80^{\circ} \mathrm{C}$ until further measurement.

2.3. Serum Aspartate Aminotransferase (AST) and Alanine Aminotransferase (ALT) Levels. The activity of AST and ALT in serum, indicators of liver cellular damage, was measured by a clinical chemistry analyzer system.

2.4. Histological Examination of Liver Sections. Median hepatic lobes were fixed in $4 \%$ buffered formalin. After embedding and cutting of $4 \mu \mathrm{m}$ slices, all samples were stained with hematoxylin/eosin. The staining sections were visualized and images were acquired using a microscope with 10x and 40x objectives. Histological evaluation was performed in a blinded manner. The severity of liver injury was graded with modified Suzuki criteria [15].

2.5. Assay of Inflammatory Cytokines and Oxidative Stress Markers. Part of the liver was homogenized with a Potter liver homogenizer at $500 \mathrm{~g}$ and centrifuged at $800 \mathrm{~g}$ for $10 \mathrm{~min}$. The supernatant was pipetted into a fresh Eppendorf cup for the detection of cytokines. Inflammatory cytokines including TNF- $\alpha$, IL- $1 \beta$, and IL- 6 in the liver were quantified with commercial ELISA kits (KeyGen BioTech, China). And the neutrophil elastase and 8-isoprostane were detected using ELISA kits (Cayman Chemical). Superoxide dismutase (SOD) and myeloperoxidase (MPO) activities were measured according to our previous methods [16].

2.6. Immunohistochemical Staining of $p 47^{p h o x}$. Immunohistochemical staining was performed in a previous study [16]. Liver tissue sections were deparaffinized, hydrated, and incubated at $4{ }^{\circ} \mathrm{C}$ overnight with a $\mathrm{p} 47^{\text {phox }}$ primary antibody (Cell Signaling Technology, Danvers, USA, diluted 1:500), followed by incubation with a horseradish peroxidasecoupled anti-rabbit IgG secondary antibody at room temperature for 2 hours and then colored with diaminobenzidine (DAB) for 3 minutes. Phosphate-buffered saline (PBS) was used to replace primary antibody in the negative control. $\mathrm{DAB}$ staining intensity was observed under light microscope (Leica Microsystems Digital Imaging, Cambridge, UK) and assessed with a microscopic image analysis system (ImageJ, National Institutes of Health, USA).

2.7. TUNEL for DNA Fragmentation. The nuclear DNA fragmentation, a specific biochemical hallmark of apoptosis, was labeled by TUNEL staining with a Dead End Fluorometric TUNEL system kit (Promega Corp., Madison, Wisconsin, USA) according to the manufacturer's protocol and our previous study [17]. Liver sections were incubated with proteinase $\mathrm{K}$ solution $(20 \mu \mathrm{g} / \mathrm{ml}$ in PBS) at room temperature for 10 minutes after deparaffinization and hydration. TUNEL labeling was conducted with a $100 \mu \mathrm{l}$ mix of equilibration buffer, nucleotide mix, and recombinant terminal 


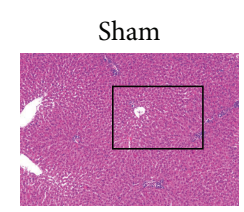

(a)

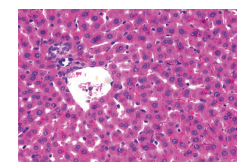

(g)

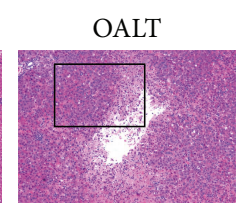

(b)

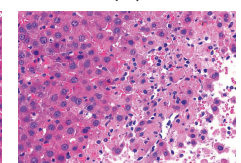

(h)

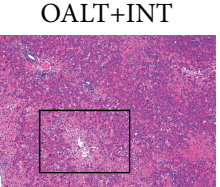

(c)

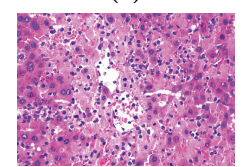

(i)

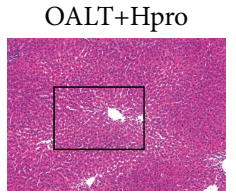

(d)

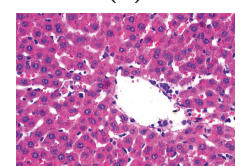

(j)

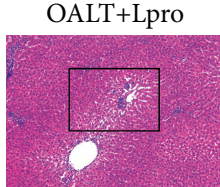

(e)

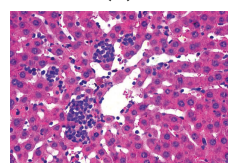

(k)
OALT+VAS

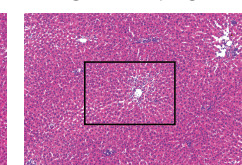

(f)

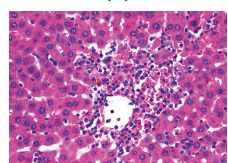

(1)

FIGURE 1: Representative photomicrographs of the livers after 8 hours of OALT. The liver tissue sections were stained with hematoxylin and eosin (H\&E staining, $100 \mathrm{x}$ and $400 \mathrm{x}$ ). $n=8$ per group. ${ }^{\#} P<0.01$ vs. the sham group; ${ }^{*} P<0.05$ and ${ }^{* *} P<0.01$ vs. the OALT group. HPro = high dose of propofol; LPro =low dose of propofol; INT = intralipid; VAS =VAS2870; OALT = orthotopic autologous liver transplantation.

deoxynucleotidyl transferase (rTdT) enzyme (the volume ratio was $45: 5: 1$ ) in a humidified, lucifugal chamber for 1 hour at $37^{\circ} \mathrm{C}$, from which step to the end of experiment, the slides were protected from direct light. The reaction was terminated by immersing the slides in a $2 \times$ SSC buffer for 15 minutes at room temperature and then were rinsed with PBS. Nuclear was visualized by DAPI staining. The liver tissues were covered by an antifade solution and mounted by glass coverslips with clear nail polish sealing the edges. The slides were immediately analyzed by a fluorescence microscope and then stored at $-20^{\circ} \mathrm{C}$ in dark if necessary.

2.8. Immunoblotting. Western blot analysis was performed in our previous studies [18]. In brief, the hepatic tissues were homogenized and nuclear proteins were extracted with a Nuclear-Cytosol Extraction kit (Applygen Technology Inc., Beijing, China) according to the manufacturer's instructions. The protein concentration had been determined by the BCA protein assay (Bio-Rad, Hemel Hempstead, Herts, UK). Sixty micrograms of each protein sample was subjected to Western blot analysis using the following primary antibodies incubated overnight at $4^{\circ} \mathrm{C}$ : anti-gp91 $1^{\text {phox }}$ at $1: 8000$ dilution (Cell Signaling Technology Inc.), anti-p47 $7^{\text {phox }}$ at $1: 1000$ dilution (Cell Signaling Technology Inc.), anti-Na/K-ATPase at 1:1000 dilution (Cell Signaling Technology Inc.), anti-cleaved caspase-3 at 1:1000 dilution (Cell Signaling Technology Inc.), antiprocaspase-3 at 1:1000 dilution (Cell Signaling Technology Inc.), anti-nuclear factor kappa B (NF- $\kappa$ B) p65 at 1:1000 dilution (Cell Signaling Technology Inc.), antiToll-like receptor 4 (TLR4) at 1:1000 dilution (Santa Cruz Biotechnology Inc.), and anti- $\beta$-actin at 1:1500 dilution (Merck Millipore, Germany). The secondary antibodies were goat anti-mouse or anti-rabbit IgG antibodies at 1 : 2000 dilution (Thermo Fisher Scientific, Fremont, CA, USA). The enhanced chemiluminescence system was used to detect the protein-antibody complex (KGP1125, Nanjing KeyGEN Biotech. Co. Ltd.). The AlphaView software (Cell Biosciences, Santa Clara, CA) was used to measure the optical density of the interesting protein band signals which were correlated to the protein levels and normalized to those of $\beta$-actin.
2.9. Analysis of Data. Data were expressed as means \pm SD. Statistical significance among groups was determined by one-way ANOVA followed by Newman-Keuls post hoc analysis using the GraphPad Prism 6 software (San Diego, CA, USA). Statistical significance was accepted at $P<0.05$.

\section{Results}

3.1. Propofol Postconditioning Reduced Liver Injury after OALT. As shown in Figure 1, compared with the sham group, there was a massive cellular necrosis (Table 1) in the centrilobular regions of the livers at 8 hours after OALT, accompanied with severe cell ballooning and infiltration of inflammatory cell, which was assessed and scaled according to the modified Suzuki criteria ( $P<0.01$ vs. the sham group). Propofol postconditioning, especially administrated at high dose $(40 \mathrm{mg} / \mathrm{kg} / \mathrm{h})$, significantly reduced the extent of necrosis, cell ballooning, and inflammatory cell infiltration $(P<0.01$ vs. the OALT group or intralipid group). Similarly, the Nox2 inhibitor VAS2870 exerted the same protective effects in the livers against I/R injury following OALT, evidenced by ameliorated cell necrosis, cell ballooning, and inflammatory cell infiltration ( $P<0.05$ vs. the OALT group). Consisted with the pathological results, as shown in Figures 2(a) and 2(b), high dose of propofol dramatically attenuated AST and ALT levels compared with the OALT group or intralipid group. These results indicated that propofol postconditioning and Nox 2 inhibition could both provide liver protection in the early stage of OALT.

3.2. Nox2 Inhibition Was Involved in the Protective Effects Conferred by Propofol Postconditioning. In order to test whether the antioxidative effect of propofol postconditioning was linked to Nox2, the Nox2 subunits p $47^{\text {phox }}$ on cell membrane and gp $91^{\text {phox }}$ in cytoplasm were detected. As shown in Figures $3(\mathrm{a})-3(\mathrm{~d})$, OALT leads to upregulation of $\mathrm{p} 47^{\text {phox }}$ and $\mathrm{gp} 91^{\text {phox }}$ protein expressions, while propofol postconditioning significantly decreased these two Nox2 subunit protein expressions in the liver following OALT. Moreover, Nox2 specific inhibitor VAS2870 was used as a positive control showing dramatically inhibition of $\mathrm{p} 47^{\text {phox }}$ and $\mathrm{gp} 91^{\text {phox }}$ protein expressions after VAS2870 treatment. 
TABLE 1: Histological score of liver injury by OALT.

\begin{tabular}{|c|c|c|c|c|c|c|c|c|c|c|c|c|c|c|c|}
\hline \multirow{3}{*}{ Groups } & \multicolumn{5}{|c|}{ Centrilobular cell death } & \multicolumn{5}{|c|}{ Ballooning } & \multicolumn{5}{|c|}{ Inflammation } \\
\hline & \multirow[b]{2}{*}{+3} & \multicolumn{3}{|c|}{ Grade } & \multirow{2}{*}{ Average } & \multicolumn{4}{|c|}{ Grade } & \multirow{2}{*}{ Average } & \multicolumn{4}{|c|}{ Grade } & \multirow{2}{*}{ Average } \\
\hline & & +2 & +1 & 0 & & +3 & +2 & +1 & 0 & & +3 & +2 & +1 & 0 & \\
\hline Sham & 0 & 0 & 0 & $8(100)$ & 0 & 0 & 0 & 0 & $8(100)$ & 0 & 0 & 0 & 0 & $8(100)$ & 0 \\
\hline OALT & $2(25)$ & $3(38)$ & $3(38)$ & $0(0)$ & $1.9^{\#}$ & $3(38)$ & $3(38)$ & $2(25)$ & $0(0)$ & $2.1^{\#}$ & $5(63)$ & $2(25)$ & $1(13)$ & $0(0)$ & $2.5^{\#}$ \\
\hline OALT + INT & $2(25)$ & $4(50)$ & $2(25)$ & $0(0)$ & $2.0^{\#}$ & $2(25)$ & $4(50)$ & $2(25)$ & $0(0)$ & $2.0^{\#}$ & $4(50)$ & $3(38)$ & $1(13)$ & $0(0)$ & $2.4^{\#}$ \\
\hline OALT + HPro & $0(0)$ & $1(13)$ & $6(75)$ & $1(13)$ & $1.0^{* *}$ & $0(0)$ & $1(13)$ & $7(88)$ & $0(0)$ & $1.1^{* *}$ & $0(0)$ & $2(25)$ & $5(63)$ & $1(13)$ & $1.1^{* *}$ \\
\hline OALT + LPro & $0(0)$ & $1(13)$ & $7(88)$ & $0(0)$ & $1.1^{* *}$ & $0(0)$ & $2(25)$ & $6(75)$ & $0(0)$ & $1.3^{*}$ & $0(0)$ & $4(50)$ & $4(50)$ & $0(0)$ & $1.5^{*}$ \\
\hline OALT + VAS & $0(0)$ & $2(25)$ & $6(75)$ & $0(0)$ & $1.3^{*}$ & $0(0)$ & $2(25)$ & $5(63)$ & $1(13)$ & $1.1^{* *}$ & $0(0)$ & $3(38)$ & $4(50)$ & $1(13)$ & $1.3^{*}$ \\
\hline
\end{tabular}

Numbers of rats are shown, with percentages enclosed within parenthesis. Grade indication: no change (0), mild (1), moderate (2), and severe (3). ${ }^{\#} P<0.01$ vs. the sham group, ${ }^{*} P<0.05$ vs. the OALT group, and ${ }^{* *} P<0.01$ vs. the OALT group.

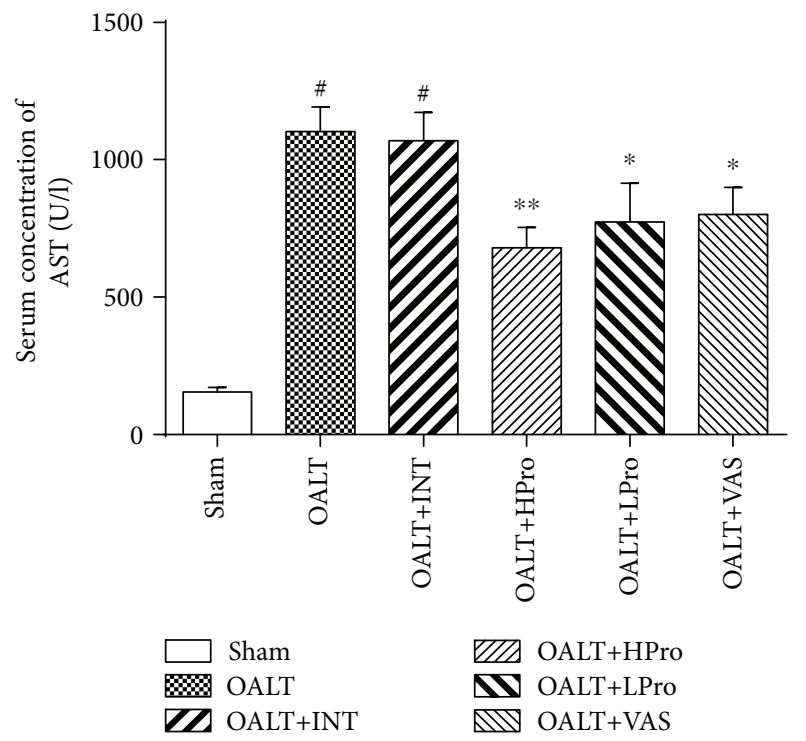

(a)
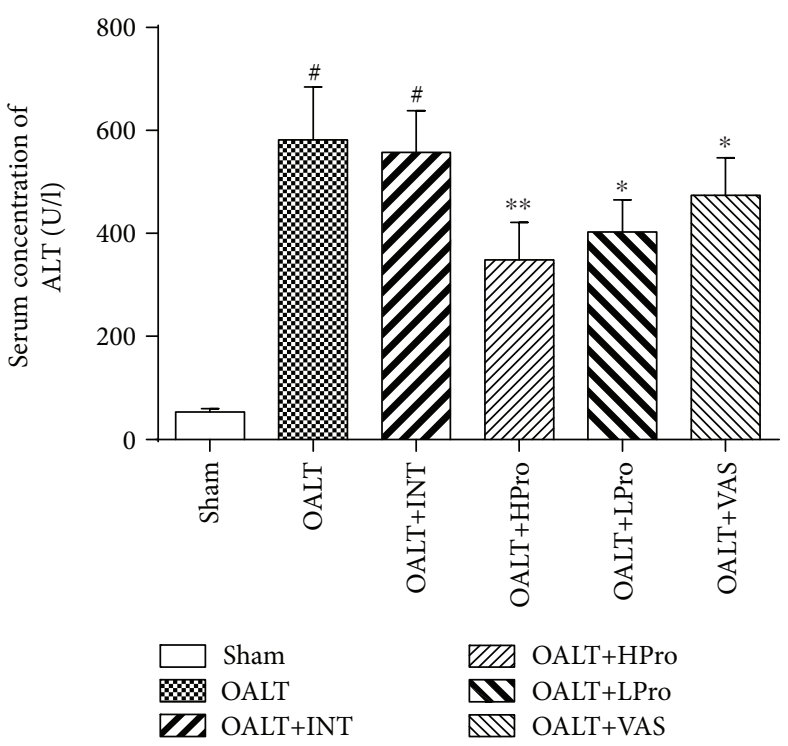

ZIA OALT+HPro

DV OALT+LPro MIV OALT+VAS

(b)

FIgURE 2: Serum alanine aminotransferase (ALT) (a) and aspartate aminotransferase (AST) (b) levels of experimental rats 8 hours after OALT. The results are expressed as the mean \pm SD. $n=8$ per group. ${ }^{\#} P<0.01$ vs. the sham group; ${ }^{*} P<0.05$ and ${ }^{* *} P<0.01$ vs. the OALT group. HPro = high dose of propofol; LPro = low dose of propofol; INT = intralipid; VAS = VAS2870; OALT = orthotopic autologous liver transplantation.

Taken together, these results revealed that propofol postconditioning may reduce hepatic oxidative stress via inhibiting NADPH oxidase Nox2 activity and the subsequent ROS generation.

\subsection{Propofol Postconditioning Attenuated Liver Inflammatory} Response following OALT. According to the pathological results, we found inflammatory infiltration during liver injury in the early stage of OALT; we then tested the inflammatory cytokines and inflammation-related TLR4/ NF- $\kappa$ B pathway. As shown in Figures 4(a)-4(c), hepatic proinflammatory cytokines TNF- $\alpha$, IL- $1 \beta$, and IL- 6 were all increased in the OALT group. Propofol postconditioning of both doses but not intralipid treatment significantly reduced the releases of proinflammatory cytokines compared to the OALT group. Treatment with Nox2 inhibitor
VAS2870 presented similar anti-inflammation effects with decrease of levels of cytokines TNF- $\alpha$, IL- $1 \beta$, and IL-6. Both propofol postconditioning and Nox 2 inhibition could inhibit the TLR $4 / \mathrm{NF}-\kappa \mathrm{B}$ inflammatory pathway, evidenced by reduced nuclear protein expressions of NF- $\kappa \mathrm{B}$ p 65 protein and downregulated total TLR4 expression (Figures 4(d)-4(f)) $(P<0.05$ vs. OALT).

3.4. Propofol Postconditioning Mitigated Neutrophil Infiltration and Hepatic Oxidative Stress. ROS scavenging is one of the characteristics of propofol. In order to detect the antioxidative effects of propofol postconditioning on liver I/R injury after OALT, neutrophil infiltration and hepatic oxidative stress were measured. As shown in Figures 5(a) and 5(b), hepatic neutrophil elastase (NE) activity and MPO activity, both of which were associated with 

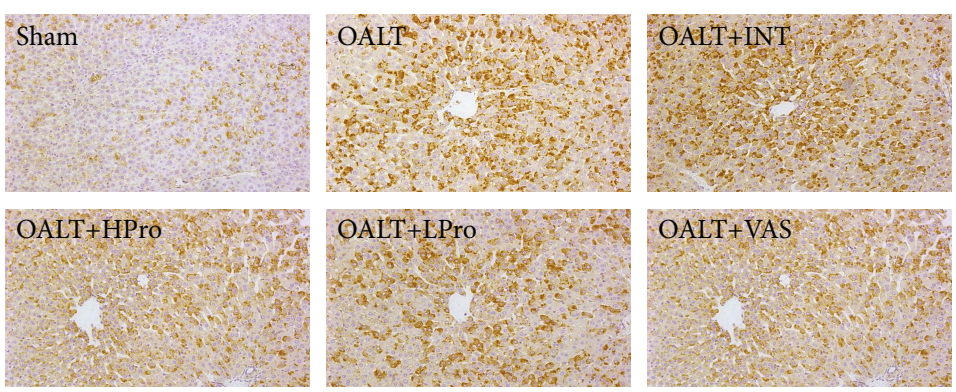

(a)

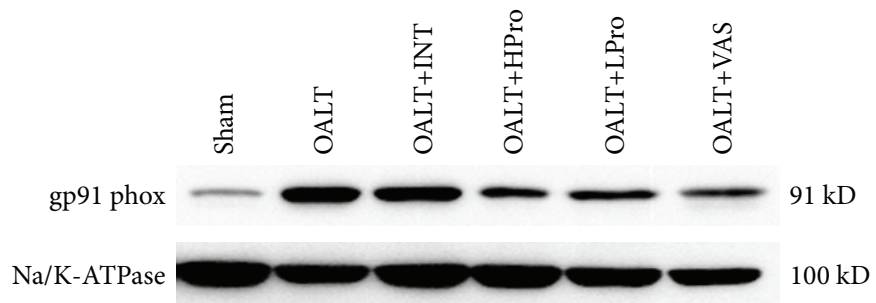

(c)

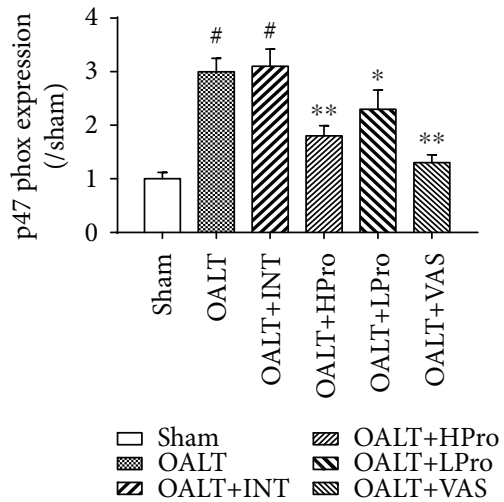

(b)

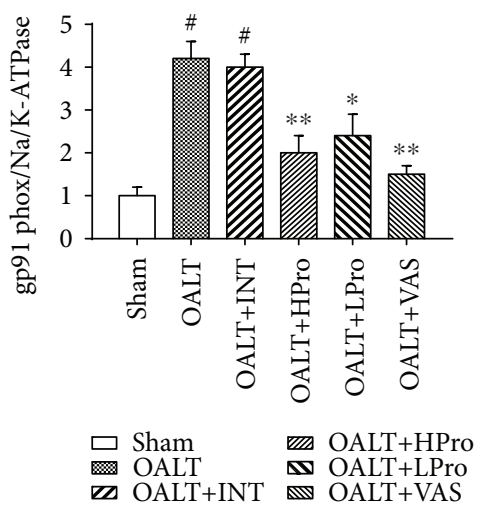

(d)

FIGURE 3: Hepatic NADPH oxidase expression changed due to OALT. Protein p $47^{\text {phox }}$ expression was detected by immunohistochemistry method (a). The amount of protein $\mathrm{p} 47^{\text {phox }}$ was calculated by gray scanning and was analyzed (b). Protein gp91 ${ }^{\text {phox }}$ expression was detected by Western blot method (c). The amount of protein gp91 ${ }^{\text {phox }}$ was calculated by gray scanning and was analyzed (d). The results are expressed as the mean \pm SD. $n=8$ per group. ${ }^{\#} P<0.01$ vs. the sham group; ${ }^{*} P<0.05$ and ${ }^{* *} P<0.01$ vs. the OALT group. HPro $=$ high dose of propofol; LPro = low dose of propofol; INT = intralipid; VAS = VAS2870; OALT = orthotopic autologous liver transplantation.

neutrophil infiltration, were significantly elevated in rats subjected to OALT. Both propofol postconditioning and VAS2870 treatment inhibited neutrophil infiltration caused by OALT and reduced lipid peroxidation product 8isoprostane generation and increased SOD activity (Figures $5(\mathrm{c})$ and $5(\mathrm{~d}))$ in the livers $(P<0.05$ vs. OALT). These results indicated that propofol postconditioning and Nox2 inhibition protected the liver from oxidative stress via reduced neutrophil infiltration and ROS generation.

3.5. Propofol Postconditioning Protected Hepatocytes from Apoptosis. Oxidative stress and inflammation can finally lead to hepatocyte apoptosis or necrosis and cause liver I/R injury. As shown in Figures 6(a)-6(c), we identified a significant amount of cell apoptosis occurred in the liver following OALT compared to the sham group $(P<0.01$ vs. the sham group). High dose of propofol postconditioning significantly reduced the number of apoptotic cells, which was consistent with the decrease of cleaved caspase-3/procaspase- 3 ratio. Similarly, Nox 2 inhibition by VAS2870 reduced hepatocyte apoptosis compared to the OALT group. These results indicated that propofol postconditioning reduced hepatocyte ROS generation and finally protected hepatocyte from apoptosis.

\section{Discussion}

In the current study, we demonstrated that propofol postconditioning reduced hepatocellular apoptosis after liver transplantation and its antioxidative property was related to inhibition of NADPH oxidase. We established an OALT model to mimic clinical liver transplantation and then detected the hepatic pathology, oxidative mediators, and inflammation response including TLR $4 / N F-\kappa B$ signaling pathway. To further clarify the protective effects of propofol, VAS2870, a specific inhibitor of NADPH oxidase Nox2, was used as a positive control. Our results suggested that propofol postconditioning exerted protective effects against liver injury following OALT. And inhibition of Nox2 maybe a possible mechanism for liver protection conferred by propofol postconditioning (Figure 7).

Liver I/R injury has been identified as one of the most important factors to the etiology after liver transplantation, which contributes to early graft dysfunction and failure [19]. $\mathrm{I} / \mathrm{R}$ processed is triggered when a donor liver is transiently deprived of oxygen and reoxygenation, leading to uncontrolled inflammatory response and reactive oxygen species release in the early stages of reperfusion [20]. In the present study, we found dramatically increased hepatic inflammatory 


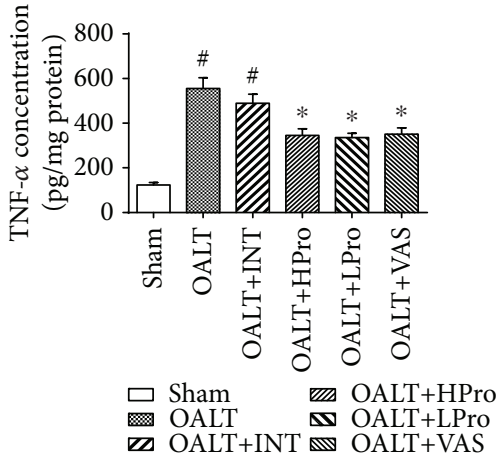

(a)

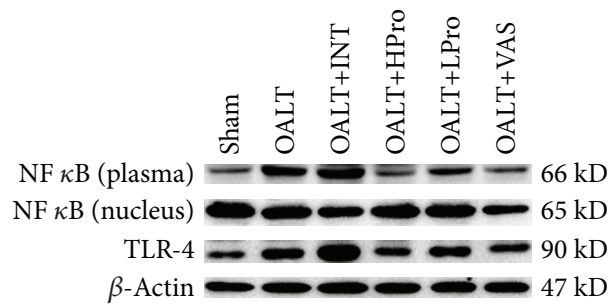

(d)

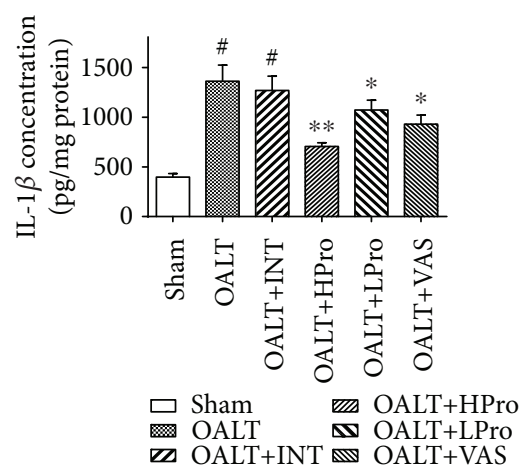

(b)

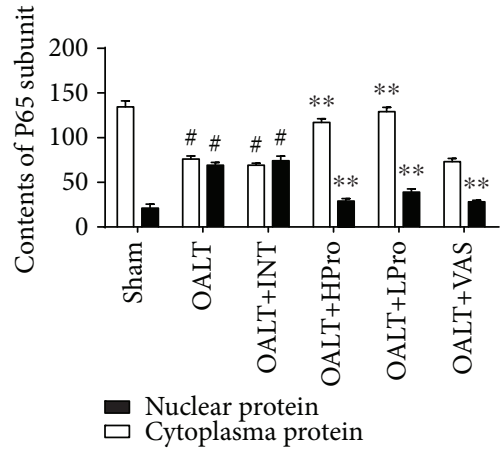

(e)

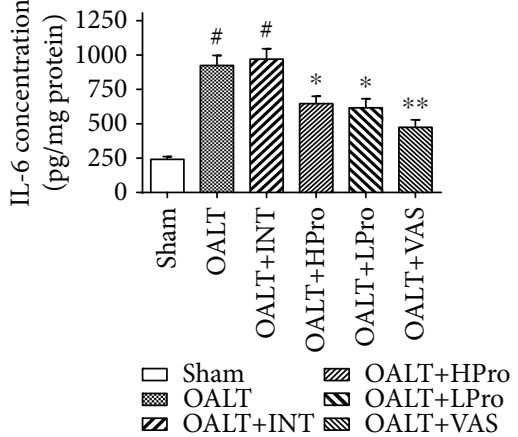

(c)

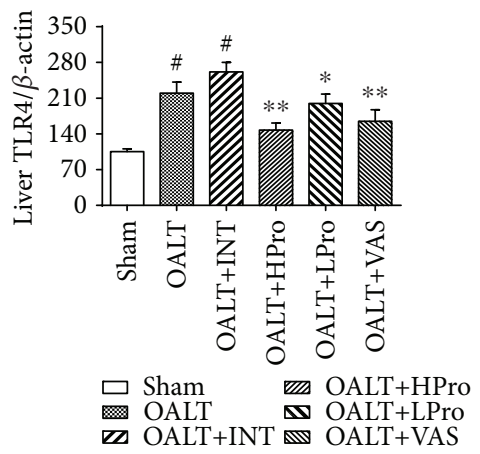

(f)

FIgURE 4: Hepatic inflammatory response after OALT. Proinflammatory cytokines TNF- $\alpha$ (a), IL- $1 \beta$ (b), and IL-6 (c) levels were measured by ELISA assay. Cytoplasm and nuclear NF- $\kappa$ B p 65 and TLR4 proteins were detected by Western blot (d). The amount of target proteins NF- $\kappa$ B p 65 (e) and TLR4 (f) was calculated by gray scanning and was analyzed. $n=8$ per group. ${ }^{\#} P<0.01$ vs. the sham group; ${ }^{*} P<0.05$ and ${ }^{* *} P<0.01$ vs. the OALT group. HPro = high dose of propofol; LPro = low dose of propofol; INT = intralipid; VAS = VAS2870; OALT = orthotopic autologous liver transplantation.

cytokines including TNF- $\alpha$, IL- $1 \beta$, and IL- 6 as well as activated TLR4/NF- $\kappa$ B signaling pathway after OALT. These cytokines were proved to be vital to the initiation and propagation of liver I/R injury, whose main role was to recruit circulating neutrophils to the injured liver tissue during reperfusion [21]. In our study, hepatic MPO and neutrophil elastase were increased, which indicated that neutrophil extensively infiltrates the liver in the early stage of OALT. Along with neutrophil infiltration was excessive ROS generation and subsequent oxygen-derived product formation [22].

Strong evidences have illustrated the importance of ROS in pathogenesis of liver I/R injury [23,24]. One of the currently promising intervention strategies is ischemic preconditioning (IPC), which is an intrinsic process whereby repeated short episode of ischemia to protect the liver against subsequent ischemia [25]. However, IPC may lead to potential vascular injury and thermogenesis [26]. We previously used propofol pretreatment during liver transplantation and found that propofol protected the lung from oxidative stress via enhancing antioxidant enzyme HO-1 expression. However, it takes several days to pretreat with propofol but most liver transplantations are emergency operations $[6,7]$. Thus, we preferred propofol postconditioning and demonstrated its protective function in reducing early liver damage after transplantation. In the model of rats' middle cerebral artery occlusion, it has proved that propofol postconditioning $(20 \mathrm{mg} / \mathrm{kg} / \mathrm{h}$ for 2 hours at the onset of reperfusion) led to long-term recovery of brain functions and upregulating the activity of the $\mathrm{PKM} \zeta / \mathrm{KCC} 2$ pathway [27]. $\mathrm{Li}$ et al. found that propofol postconditioning enhanced cell viability and alleviated apoptosis to protect cardiomyocytes against hypoxia/reoxygenation injury through ERK signaling pathway [28]. Of interest, $\mathrm{Li}$ et al. found that alternative use of isoflurane and propofol conferred superior cardioprotection against postischemic myocardial injury and dysfunction, and this function was probably mediated through attenuating cardiac oxidative damage [29], which indicated that anesthesia may play an important role in organ protection during I/R injury.

NADPH oxidase activation and subsequent ROS formation are important upstream events which can activate hepatocytes and amplify the production of multiple proinflammatory cytokines, such as TNF- $\alpha$ or interlukin-1 $\beta$ [30]. Hepatic NADPH oxidase activation and the ROS production have been implicated as critical regulators of liver I/R injury [23]. Although propofol has been shown to reduce oxidative stress 


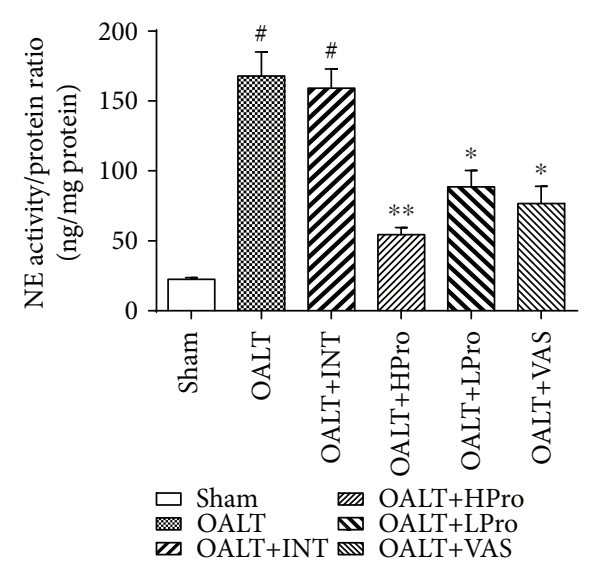

(a)

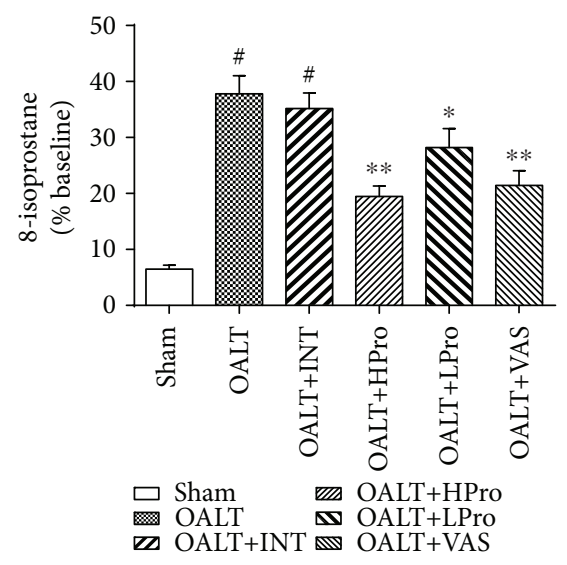

(c)

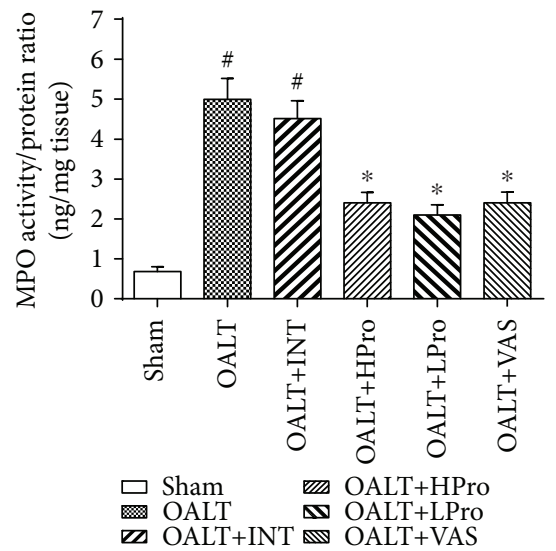

(b)

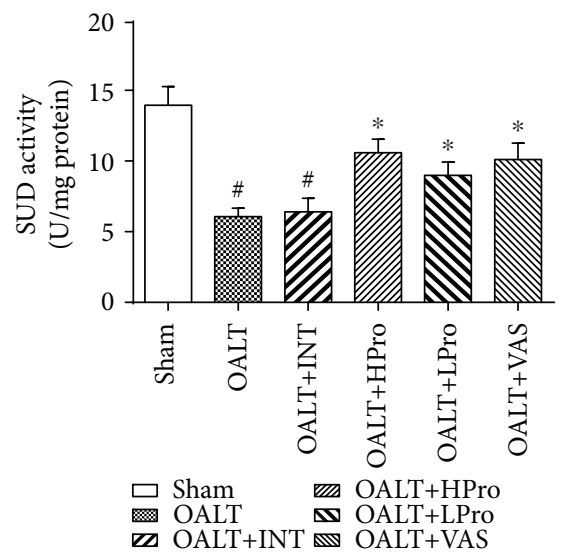

(d)

FIGURE 5: Neutrophil infiltration and oxidative stress in the liver. Neutrophil elastase (NE) (a) and myeloperoxidase (MPO) (b) activities reflected neutrophil infiltration. 8-Isoprostane (c) and superoxide dismutase (SOD) (d) were detected to reflect hepatic oxidative stress level. $n=8$ per group. ${ }^{\#} P<0.01$ vs. the sham group; ${ }^{*} P<0.05$ and ${ }^{* *} P<0.01$ vs. the OALT group. HPro $=$ high dose of propofol; LPro = low dose of propofol; INT $=$ intralipid; VAS $=$ VAS2870; OALT $=$ orthotopic autologous liver transplantation.

as an ROS scavenger, the current study shows that propofol can also suppress Nox 2 to reduce the consequent production of ROS. Notably, propofol downregulated the hepatic expression of the NADPH oxidase membrane components $\mathrm{p} 47^{\text {phox }}$ and glycosylated subunit gp91 ${ }^{\text {phox }}$ after OALT. We speculated that this may be an important mechanism of propofol actions. Luo et al. showed that siRNA silencing of $\mathrm{p} 22^{\text {phox }}$ significantly attenuated the protective effects of propofol [31]. Recent studies have also identified other receptors as potential molecular targets of propofol including nicotinic and M1 muscarinic receptors $[32,33]$. Whether these receptors act as upstream regulators, NADPH oxidase remains to be determined.

Of note, in the current study, the OALT model is superior in mimicking the pathophysiological variation during liver ischemia/reperfusion in liver transplantation and ischemia/ reperfusion-mediated liver injury without interference of immunoactivities between grafts and hosts. However, as the cold ischemia time in this model is about 20 minutes, so it is not able to represent the long (6 to 8 hours) cold preservation time that occurs in the liver graft before being transplanted. Propofol postconditioning was performed at the onset of reperfusion with continuous infusion for $30 \mathrm{~min}$ and proved to be protective against liver I/R injury. However, whether the dose and the duration we chose was the best intervention required further investigation. Moreover, although we have confirmed that the protective effect of propofol postconditioning was related to Nox2 activity inhibition, whether it acts on the other NADPH oxidase subunit such as Nox4 also remains unknown. It is also still unclear that how propofol acts on Nox2, directly or indirectly. Those questions remain unanswered. More studies will be involved to clarify these mechanisms and make this intervention more safe and reliable.

In summary, liver transplantation leads to severe inflammation and oxidative stress accompanied with NADPH oxidase Nox2 activation. Propofol postconditioning exerted prominently protective function against the I/R injury after liver transplantation, which presented as lower levels of inflammatory mediators and oxidative products accompanied with less neutrophil filtration and weaker induction of Nox2. Collectively, propofol postconditioning had been proved to reduce liver inflammation and oxidative stress probably via inhibiting NADPH oxidase Nox2. 

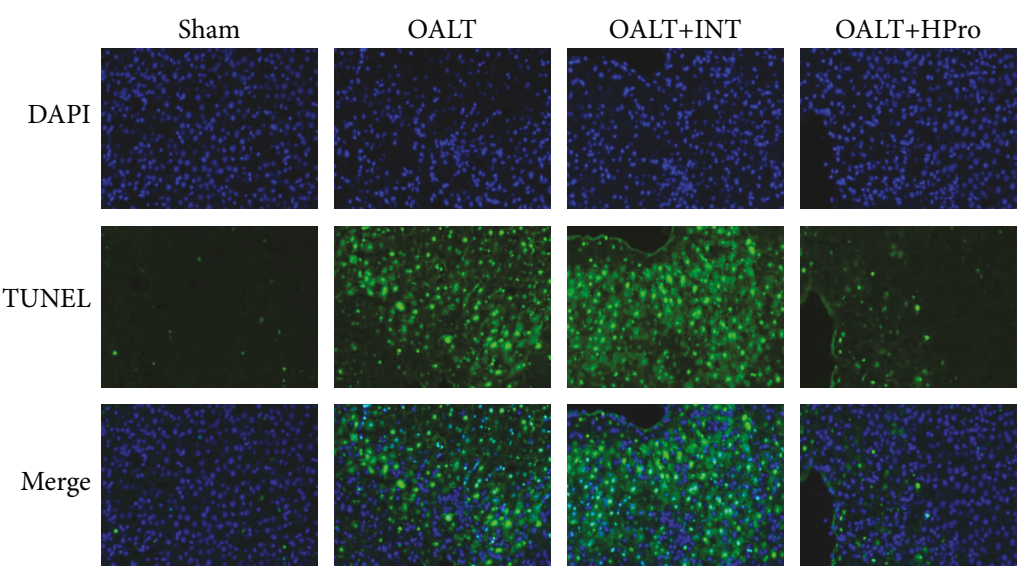

OALT+LPro
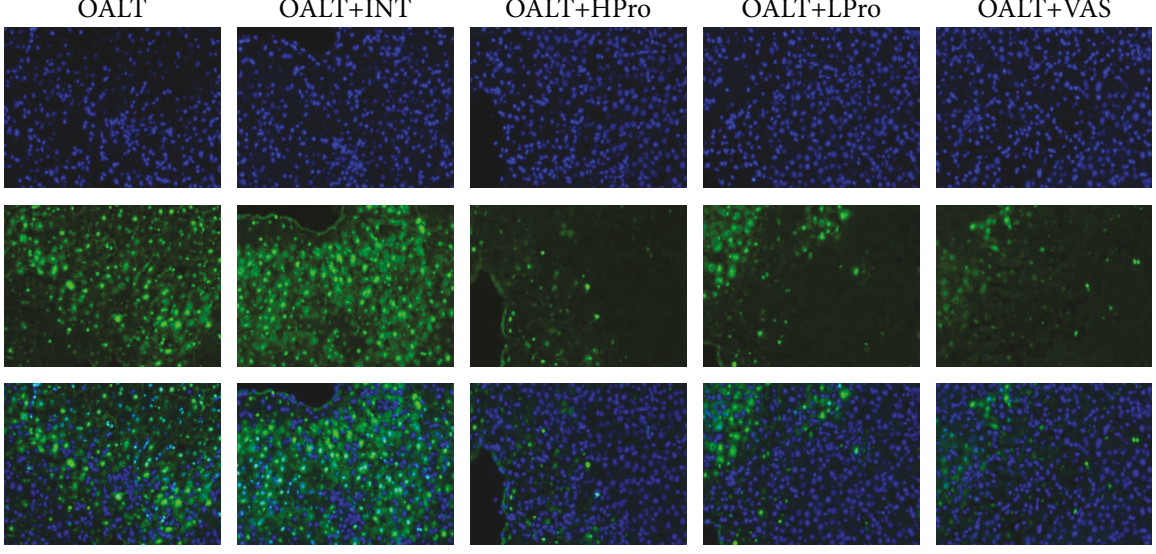

(a)
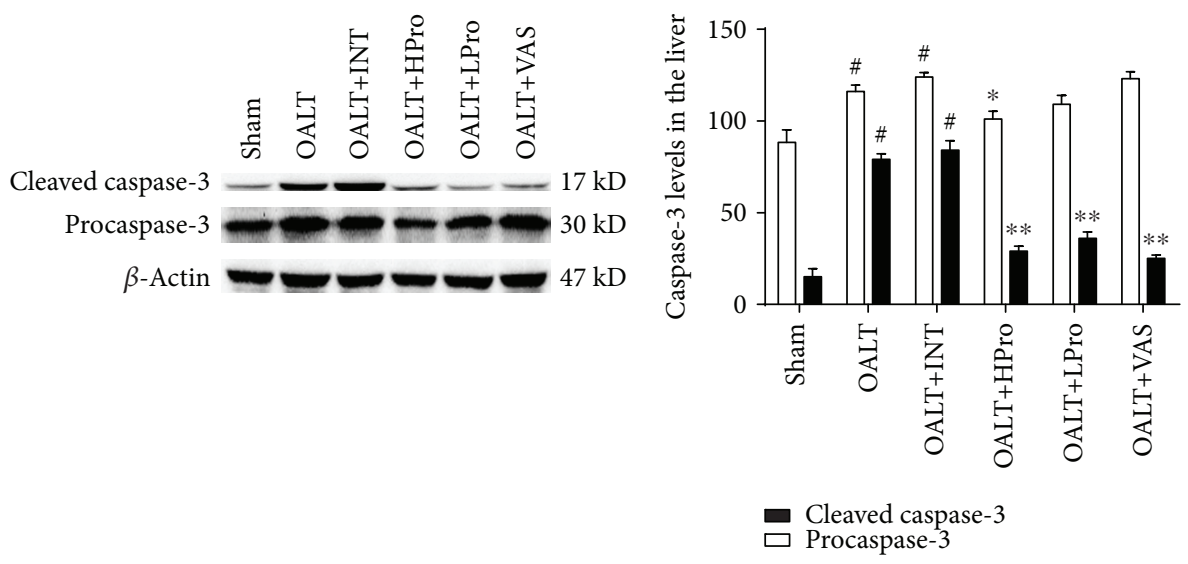

(b)

(c)

Figure 6: Propofol postconditioning protected hepatocyte from apoptosis. Fluorescent TUNEL staining of liver tissue (40x) (a). Cleaved caspase-3 and procaspase-3 proteins were detected by Western blot method (b). The amount of target proteins cleaved caspase- 3 and procaspase- 3 was calculated by gray scanning and was analyzed (c). The results are expressed as the mean \pm SD. $n=8$ per group. ${ }^{\#} P<0.01$ vs. the sham group; ${ }^{*} P<0.05$ and ${ }^{* *} P<0.01$ vs. the OALT group. HPro $=$ high dose of propofol; LPro $=$ low dose of propofol; INT = intralipid; VAS = VAS2870; OALT $=$ orthotopic autologous liver transplantation.

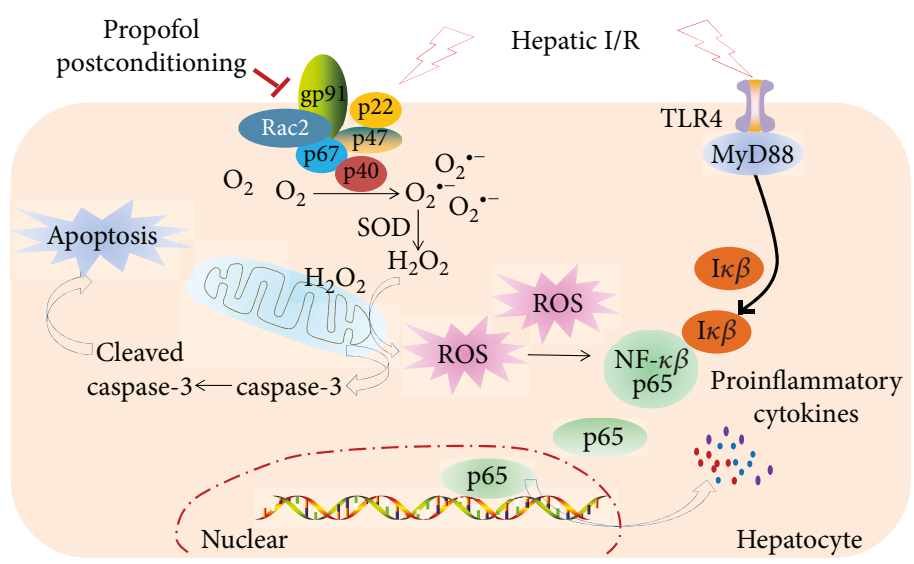

FIGURE 7: Propofol postconditioning reduces liver injury and the possible mechanisms. Under hepatic I/R condition, NADPH oxidase and TLR4/NF- $\kappa$ B pathway are activated. Endogenously, ROS were generated due to NADPH oxidase activation, resulting in the caspase-3related apoptosis pathway as well as NF- $\kappa \mathrm{B}$ pathway activation. Amount of proinflammatory cytokines was produced after NF- $\kappa \mathrm{B}$ p65 pathway activation. Propofol postconditioning inhibited Nox $2\left(\mathrm{gp} 91^{\mathrm{phox}}\right.$ and $\mathrm{p} 47^{\text {phox }}$ ) which could lead to downregulation of ROS generation and finally reduced hepatic I/R injury. 


\section{Data Availability}

The data used to support the findings of this study are available from the corresponding author upon request.

\section{Conflicts of Interest}

The authors declare that they have no competing interests.

\section{Authors' Contributions}

Weifeng Yao and Xue Han contributed equally to this study. Weifeng Yao, Ziqing Hei, and Pinjie Huang conceived and designed the experiments. Weifeng Yao, Xue Han, Yihan Zhang, Jianqiang Guan, Mian Ge, Chaojin Chen, Shan Wu, Jiaxin Chen, Gangjian Luo, and Pinjie Huang performed the experiments. Weifeng Yao analyzed the data. Xue Han contributed the reagents/materials/analysis tools. Weifeng Yao wrote the paper. All authors read and approved the manuscript.

\section{Acknowledgments}

The study was supported in part by grants from the National Natural Science Foundation of China (no. 81601724 for Weifeng Yao; no. 81501693 for Pinjie Huang and no. 81770649 for Gangjian Luo; no. 81772127 for Ziqing Hei). The study also was supported in part by grants from the Natural Science Foundation of Guangdong Province (no. 2016A030313232 for Pinjie Huang).

\section{References}

[1] F. Durand, "How to improve long-term outcome after liver transplantation?," Liver International, vol. 38, Supplement 1, pp. 134-138, 2018.

[2] R. F. Saidi and S. K. H. Kenari, "Liver ischemia/reperfusion injury: an overview," Journal of Investigative Surgery, vol. 27, no. 6, pp. 366-379, 2014.

[3] J. Li, R. J. Li, G. Y. Lv, and H. Q. Liu, "The mechanisms and strategies to protect from hepatic ischemia-reperfusion injury," European Review for Medical and Pharmacological Sciences, vol. 19, no. 11, pp. 2036-2047, 2015.

[4] P. Tang and R. Eckenhoff, "Recent progress on the molecular pharmacology of propofol," F1000Res, vol. 7, p. 123, 2018.

[5] I. Vasileiou, T. Xanthos, E. Koudouna et al., "Propofol: a review of its non-anaesthetic effects," European Journal of Pharmacology, vol. 605, no. 1-3, pp. 1-8, 2009.

[6] M. Ge, W. Yao, Y. Wang et al., "Propofol alleviates liver oxidative stress via activating Nrf2 pathway," The Journal of Surgical Research, vol. 196, no. 2, pp. 373-381, 2015.

[7] W. Yao, G. Luo, G. Zhu et al., "Propofol activation of the Nrf2 pathway is associated with amelioration of acute lung injury in a rat liver transplantation model," Oxidative Medicine and Cellular Longevity, vol. 2014, Article ID 258567, 9 pages, 2014.

[8] D. Bracco, "Post-conditioning: promising answers and more questions," Critical Care, vol. 16, no. 6, p. 180, 2012.

[9] H. Harada, I. N. Hines, S. Flores et al., "Role of NADPH oxidase-derived superoxide in reduced size liver ischemia and reperfusion injury," Archives of Biochemistry and Biophysics, vol. 423, no. 1, pp. 103-108, 2004.
[10] S. H. Ellmark, G. J. Dusting, M. N. T. Fui, N. Guzzo-Pernell, and G. R. Drummond, "The contribution of Nox4 to NADPH oxidase activity in mouse vascular smooth muscle," Cardiovascular Research, vol. 65, no. 2, pp. 495-504, 2005.

[11] W. Yao, H. Li, G. Luo et al., "SERPINB1 ameliorates acute lung injury in liver transplantation through ERK1/2-mediated STAT3-dependent HO-1 induction," Free Radical Biology \& Medicine, vol. 108, pp. 542-553, 2017.

[12] C. Luo, D. Yuan, X. Li et al., "Propofol attenuated acute kidney injury after orthotopic liver transplantation via inhibiting gap junction composed of connexin 32," Anesthesiology, vol. 122, no. 1, pp. 72-86, 2015.

[13] A. G. Hudetz, X. Liu, S. Pillay, M. Boly, and G. Tononi, "Propofol anesthesia reduces Lempel-Ziv complexity of spontaneous brain activity in rats," Neuroscience Letters, vol. 628, pp. 132-135, 2016.

[14] Y. H. Tuo, Z. Liu, J. W. Chen et al., "NADPH oxidase inhibitor improves outcome of mechanical reperfusion by suppressing hemorrhagic transformation," Journal of NeuroInterventional Surgery, vol. 9, no. 5, pp. 492-498, 2017.

[15] A. Naiki-Ito, M. Asamoto, T. Naiki et al., “Gap junction dysfunction reduces acetaminophen hepatotoxicity with impact on apoptotic signaling and connexin 43 protein induction in rat," Toxicologic Pathology, vol. 38, no. 2, pp. 280-286, 2010.

[16] X. Huang, W. Zhao, D. Hu et al., "Resveratrol efficiently improves pulmonary function via stabilizing mast cells in a rat intestinal injury model," Life Sciences, vol. 185, pp. 30-37, 2017.

[17] W. Yao, H. Li, X. Han et al., "MG53 anchored by dysferlin to cell membrane reduces hepatocyte apoptosis which induced by ischaemia/reperfusion injury in vivo and in vitro," Journal of Cellular and Molecular Medicine, vol. 21, no. 10, pp. 25032513, 2017.

[18] M. Ge, W. Yao, D. Yuan et al., "Brg1-mediated Nrf2/HO-1 pathway activation alleviates hepatic ischemia-reperfusion injury," Cell Death \& Disease, vol. 8, no. 6, article e2841, 2017.

[19] M. Mendes-Braz, M. Elias-Miro, M. B. Jimenez-Castro, A. Casillas-Ramirez, F. S. Ramalho, and C. Peralta, "The current state of knowledge of hepatic ischemia-reperfusion injury based on its study in experimental models," Journal of Biomedicine \& Biotechnology, vol. 2012, Article ID 298657, 20 pages, 2012.

[20] M. Abu-Amara, S. Y. Yang, N. Tapuria, B. Fuller, B. Davidson, and A. Seifalian, "Liver ischemia/reperfusion injury: processes in inflammatory networks-a review," Liver Transplantation, vol. 16, no. 9, pp. 1016-1032, 2010.

[21] K. M. Quesnelle, P. V. Bystrom, and L. H. Toledo-Pereyra, "Molecular responses to ischemia and reperfusion in the liver," Archives of Toxicology, vol. 89, no. 5, pp. 651-657, 2015.

[22] H. Jaeschke, "Reactive oxygen and mechanisms of inflammatory liver injury: present concepts," Journal of Gastroenterology and Hepatology, vol. 26, Suppl 1, pp. 173-179, 2011.

[23] H. Jaeschke and B. L. Woolbright, "Current strategies to minimize hepatic ischemia-reperfusion injury by targeting reactive oxygen species," Transplantation Reviews, vol. 26, no. 2, pp. 103-114, 2012.

[24] H. Jaeschke, "Mechanisms of liver injury. II. Mechanisms of neutrophil-induced liver cell injury during hepatic ischemiareperfusion and other acute inflammatory conditions," 
American Journal of Physiology-Gastrointestinal and Liver Physiology, vol. 290, no. 6, pp. G1083-G1088, 2006.

[25] S. Yan, L. M. Jin, Y. X. Liu, L. Zhou, H. Y. Xie, and S. S. Zheng, "Outcomes and mechanisms of ischemic preconditioning in liver transplantation," Hepatobiliary \& Pancreatic Diseases International, vol. 9, no. 4, pp. 346-354, 2010.

[26] R. S. Koti, A. M. Seifalian, and B. R. Davidson, "Protection of the liver by ischemic preconditioning: a review of mechanisms and clinical applications," Digestive Surgery, vol. 20, no. 5, pp. 383-396, 2003.

[27] C. Y. Yang, S. Y. Liu, H. Y. Wang et al., "Neuroprotection by propofol post-conditioning: focus on $\mathrm{PKM} \zeta / \mathrm{KCC} 2$ pathway activity," Cellular and Molecular Neurobiology, vol. 38, no. 3, pp. 691-701, 2018.

[28] H. Li, J. Tan, Z. Zou, C. G. Huang, and X. Y. Shi, "Propofol post-conditioning protects against cardiomyocyte apoptosis in hypoxia/reoxygenation injury by suppressing nuclear factor-kappa B translocation via extracellular signal-regulated kinase mitogen-activated protein kinase pathway," European Journal of Anaesthesiology, vol. 28, no. 7, pp. 525-534, 2011.

[29] T. Li, W. Wu, Z. You et al., "Alternative use of isoflurane and propofol confers superior cardioprotection than using one of them alone in a dog model of cardiopulmonary bypass," European Journal of Pharmacology, vol. 677, no. 1-3, pp. 138-146, 2012.

[30] K. Kimura, K. Shirabe, T. Yoshizumi et al., "Ischemia-reperfusion injury in fatty liver is mediated by activated NADPH oxidase 2 in rats," Transplantation, vol. 100, no. 4, pp. 791-800, 2016.

[31] T. Luo, J. Wu, S. V. Kabadi et al., "Propofol limits microglial activation after experimental brain trauma through inhibition of nicotinamide adenine dinucleotide phosphate oxidase," Anesthesiology, vol. 119, no. 6, pp. 1370-1388, 2013.

[32] S. S. Jayakar, W. P. Dailey, R. G. Eckenhoff, and J. B. Cohen, "Identification of propofol binding sites in a nicotinic acetylcholine receptor with a photoreactive propofol analog," The Journal of Biological Chemistry, vol. 288, no. 9, pp. 61786189, 2013.

[33] O. Murasaki, M. Kaibara, Y. Nagase et al., "Site of action of the general anesthetic propofol in muscarinic M1 receptormediated signal transduction," The Journal of Pharmacology and Experimental Therapeutics, vol. 307, no. 3, pp. 995-1000, 2003. 


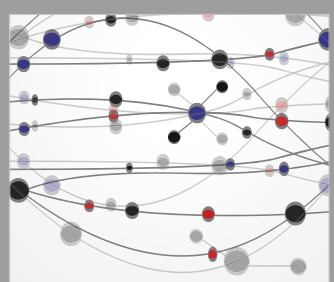

The Scientific World Journal
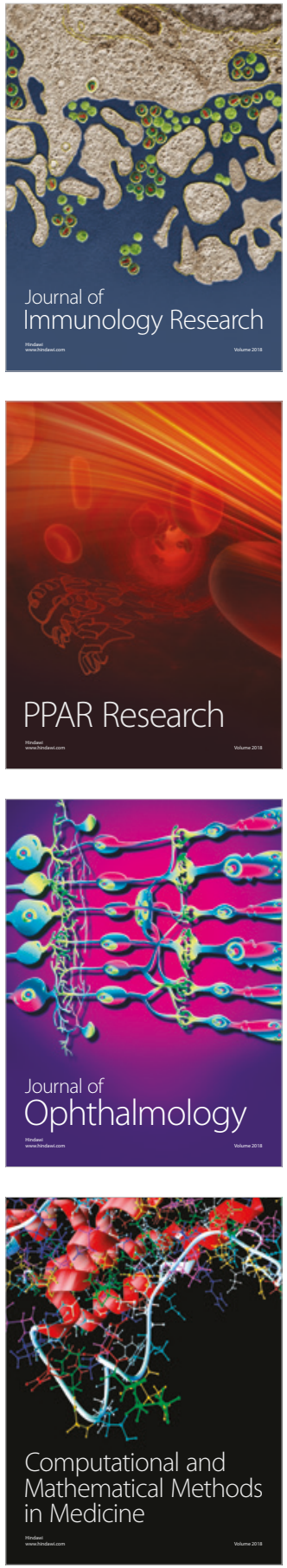

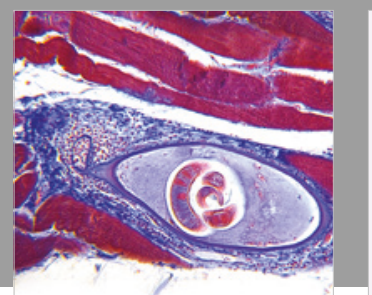

Gastroenterology Research and Practice

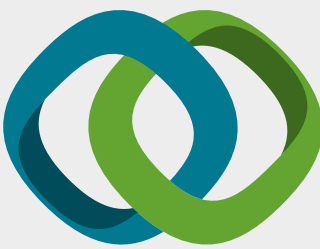

\section{Hindawi}

Submit your manuscripts at

www.hindawi.com
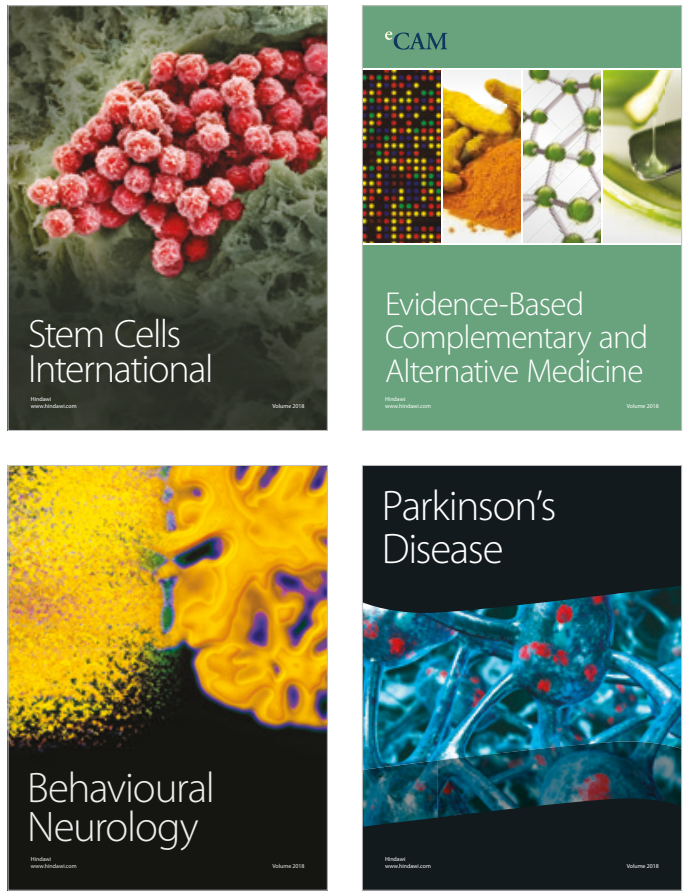

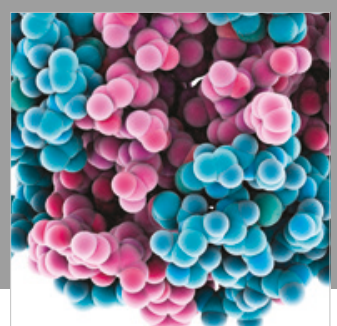

ournal of

Diabetes Research

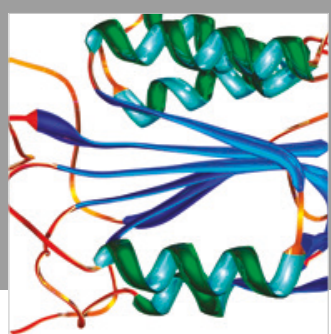

Disease Markers
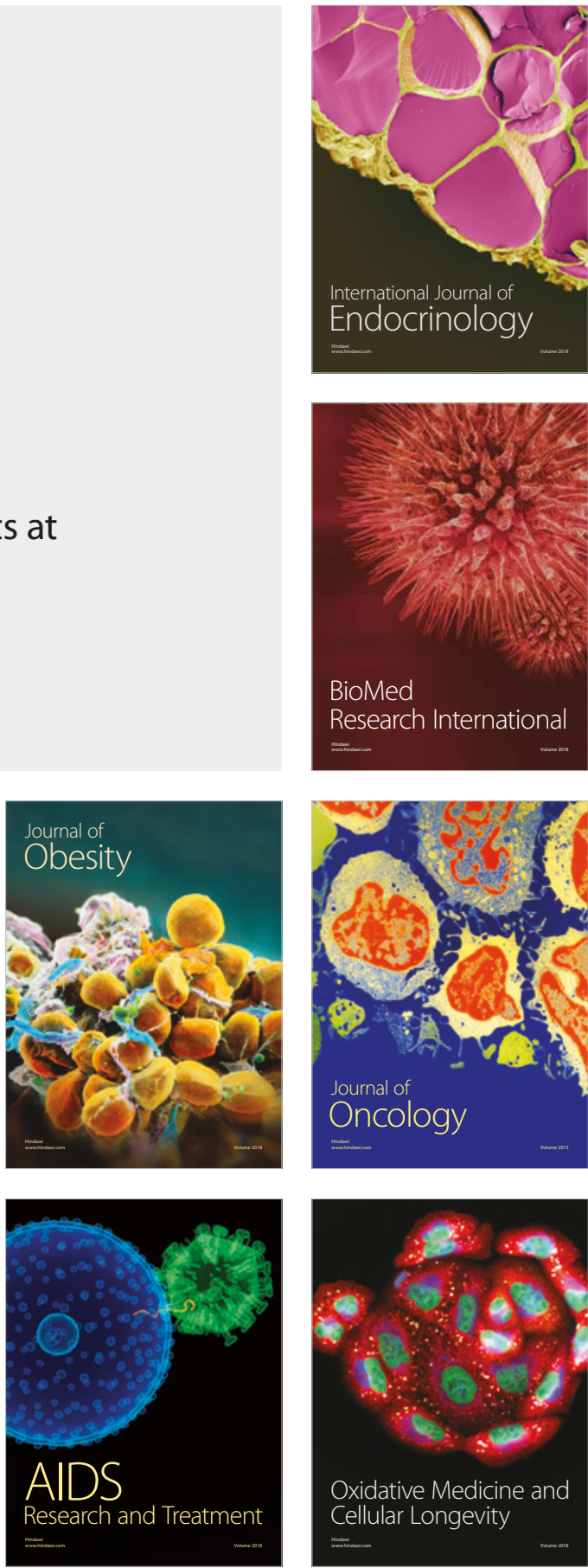